\title{
Preparation and characterization of form-stable paraffin/polycaprolactone composites as phase change materials for thermal energy storage
}

\author{
M.S. Aludin ${ }^{1, *}$, and S. Saidatul Akmal ${ }^{1}$ \\ ${ }^{1}$ Faculty of Engineering Technology, Universiti Teknikal Malaysia Melaka, Hang Tuah Jaya, 76100, \\ Durian Tunggal, Melaka, Malaysia.
}

\begin{abstract}
Paraffin is Phase Change Materials (PCM) that possesses desirable properties such as high thermal energy storage and thermal stability to make it suitable for thermal energy storage applications. However, paraffin has been reported to leak out during the melting process. In this study, composites were prepared by dissolving paraffin and polycaprolactone (PCL) at varied mass percent compositions in chloroform and then purified through precipitation techniques. The leakage test was conducted by placing the composite samples on a set of four-layer filter papers and left in a furnace at $90^{\circ} \mathrm{C}$ for 1 hour. By incorporating PCL into paraffin phase, the leakage mass percentage was drastically reduced. The PCL polymer matrix in the composites may have trapped the paraffin molecules during melting process thus prevent it from leaking.
\end{abstract}

\section{Introduction}

Thermal energy storage (TES) functions as temporary storage of thermal energy in the forms of cold or heat energy. TES can be generally classified as sensible heat storage and latent heat storage [1]. Latent heat storage is the most preferred forms of energy storage because it can provide high energy storage density and nearly isothermal heat storage or retrieval processes [2]. The latent heat can be discharged or charged based on demand and applications. One of prospective techniques of storing thermal energy is the application of phase change materials (PCM). PCM are latent heat storage materials that can absorb, store and release latent heat of fusion to the environment during melting and freezing processes [3]. Solid-liquid phase change process is illustrated in Fig. 1.

* Corresponding author: aludin@utem.edu.my 


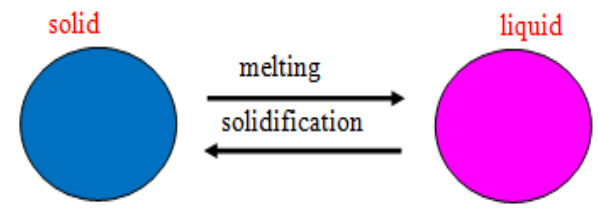

Fig. 1. Solid-liquid phase change process

Paraffin (saturated hydrocarbons with $\mathrm{C}_{\mathrm{n}} \mathrm{H}_{2 \mathrm{n}+2}$ formulas) not only can absorb and store heat but it also can release large amount of heat over a large number of phase change cycle which make it useful as an excellent PCM [4]. Paraffin is also considered non-toxic. However, the major problem of using paraffin is leakage during melting process $[5,6]$. As a result, in recent years, much attention has been paid to form-stable paraffin PCM which consists of solid-liquid paraffin and matrix that provides supportive function [2]. One of the selected matrixes is polymer. In this study, paraffin/polycaprolactone (PCL) composites were prepared at varied mass percent compositions in order to obtain form-stable PCM composites thus possibly eliminates the leakage problem. PCL was selected as the polymer matrix since it has good mechanical properties and excellent miscibility with a large range of materials and it is one of the few commercially available biodegradable polymers [7].

\section{Methodology}

\subsection{Materials}

Technical grade paraffin wax with melting temperature $56^{\circ} \mathrm{C}$, supplied by Aldrich Co., Inc. PCL with average molecular weight 80,000 and melting temperature $61^{\circ} \mathrm{C}$ was also purchased from Aldrich Co., Inc. Chloroform and ethanol were purchased from Kanto Chemical Co., Inc. Both of the chemicals had a purity of $99 \mathrm{wt} \%$ and were used as received.

\subsection{Preparation of paraffin/PCL composites}

The composites were prepared by dissolving paraffin and PCL at varied mass percent compositions in chloroform and then purified through precipitation techniques by using ethanol solution. The composites were then dried in a fume hood.

\subsection{Thermal properties of paraffin/PCL composites}

The melting temperature and the latent heat of fusion of the composites were measured by Differential Scanning Calorimeter (DSC). The DSC measurements were performed in the temperature range of 0 to $80^{\circ} \mathrm{C}$ with a heating rate $10^{\circ} \mathrm{C}$ per minute and under a constant stream of nitrogen at atmospheric pressure.

\subsection{Leakage test}

The leakage test was conducted by placing $1.0 \mathrm{~g}$ of each composite sample on a set of fourlayer filter papers and left in a furnace at $90^{\circ} \mathrm{C}$ for 1 hour. Leakage test set-up in a furnace is illustrated in Fig. 2. The leakage percentage was calculated based on the change of sample mass before and after the leakage test. 


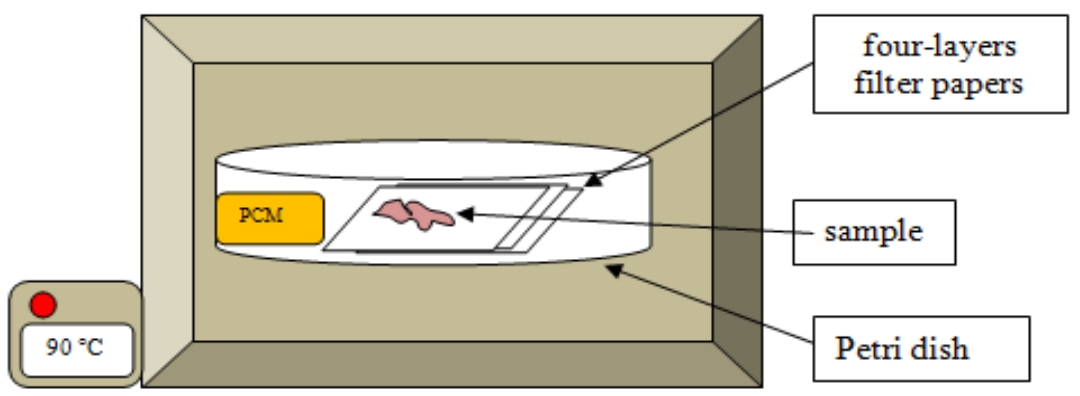

Fig. 2. Leakage test set-up

\section{Results and discussions}

The leakage test results show that the leakage mass percentage of the composites is significantly lower than that of pure paraffin (leakage percentage 78.4\%). The composite with percentage mass composition of paraffin/PCL 40/60 shows almost no leakage during the leakage test. Table 1 tabulates the overall leakage test results.

Table 1. Leakage test results

\begin{tabular}{|c|c|c|c|}
\hline Sample No. & \multirow{2}{*}{$\begin{array}{c}\text { Paraffin / PCL } \\
\text { (Mass\%) }\end{array}$} & \multicolumn{2}{|c|}{ Leakage Mass } \\
\cline { 3 - 4 } & & $(\mathrm{g})$ & $(\%)$ \\
\hline PCM1 & $100 / 0$ & 0.784 & 78.4 \\
\hline PCM2 & $80 / 20$ & 0.251 & 35.1 \\
\hline PCM3 & $60 / 40$ & 0.197 & 29.7 \\
\hline PCM4 & $50 / 50$ & 0.305 & 30.5 \\
\hline PCM5 & $40 / 60$ & 0.001 & 0.10 \\
\hline PCM6 & $20 / 80$ & 0.182 & 18.2 \\
\hline PCL & $0 / 100$ & 0.000 & 0.00 \\
\hline
\end{tabular}

The resistance of PCL polymer matrix to higher temperature than the melting temperature of the paraffin makes the composites able to maintain the solid morphology in macro level even the paraffin has melted. The photographs indicating the remaining PCM on the filter papers after the leakage tests are shown in Fig. 3. As can clearly be seen in the photos, the composite sample PCM5 maintains it shape in the solid state without seepage. 

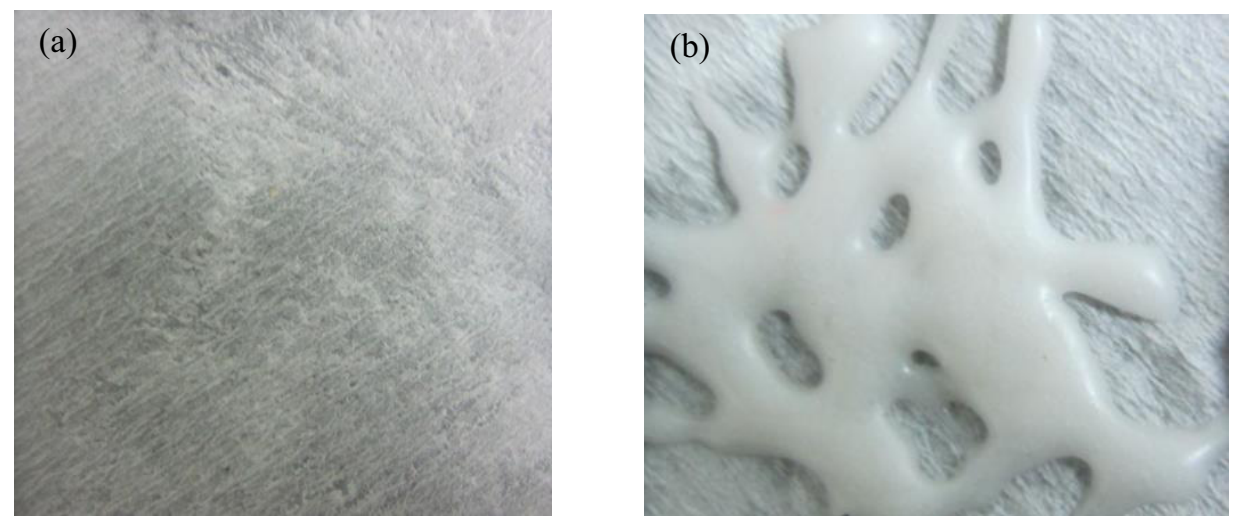

Fig. 3. Sample appearance after leakage test:
(a) PCM1 (b) PCM5

The melting temperature and latent heat of fusion of the composites that were measured by DSC are shown in Table 2 . The latent heat of fusion of the composites decreased at PCL mass compositions $40 \%$ to $60 \%$. The melting temperatures are also higher by about $4^{\circ} \mathrm{C}$ at this range of mass compositions. Fig. 4 shows the DSC thermograph of PCM5 (paraffin 40\%/PCL 60\%).

Table 2. DSC results of paraffin/PCL composites

\begin{tabular}{|c|c|c|c|}
\hline $\begin{array}{c}\text { Sample } \\
\text { No. }\end{array}$ & $\begin{array}{c}\text { Paraffin/PCL } \\
\text { (Mass \%) }\end{array}$ & $\begin{array}{c}\text { Melting } \\
\text { temperature }\left({ }^{\circ} \mathbf{C}\right)\end{array}$ & $\begin{array}{c}\text { Latent heat of fusion } \\
(\mathbf{k J} / \mathbf{k g})\end{array}$ \\
\hline PCM1 & $100 / 0$ & 55.6 & 139.2 \\
\hline PCM2 & $80 / 20$ & 56.6 & 129.1 \\
\hline PCM3 & $60 / 40$ & 64.8 & 85.2 \\
\hline PCM4 & $50 / 50$ & 63.6 & 84.3 \\
\hline PCM5 & $40 / 60$ & 65.2 & 81.4 \\
\hline PCM6 & $20 / 80$ & 56.5 & 96.7 \\
\hline PCL & $0 / 100$ & 60.6 & 72.1 \\
\hline
\end{tabular}

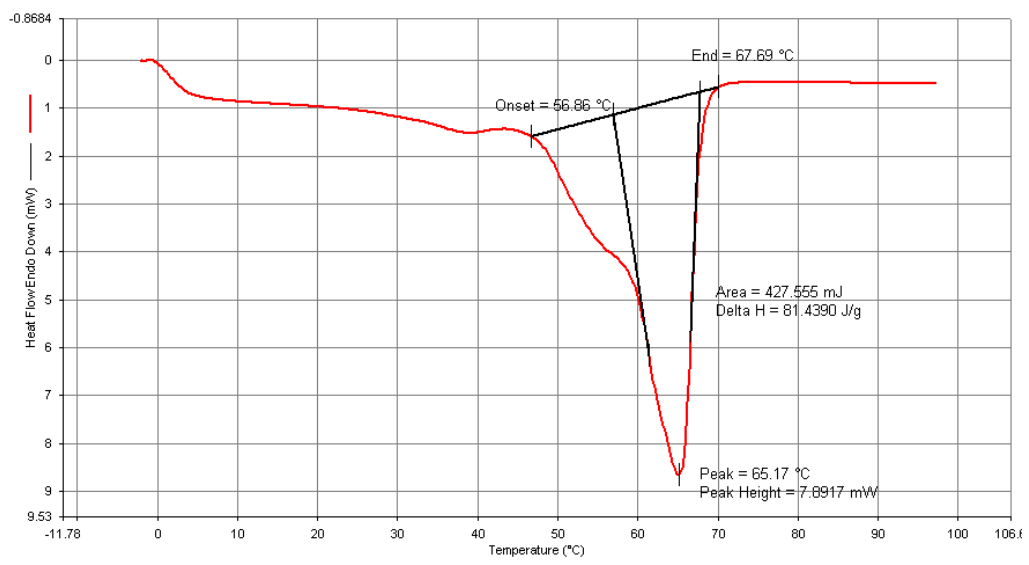

Fig. 4. DSC thermograph of PCM5 
The lower of heat of fusion and the slight increase in the melting temperatures of the composites with PCL mass compositions $40 \%$ to $60 \%$ indicated that paraffin phase and PCL matrix phase were homogeneously mixed. Therefore, the PCL polymer matrix in the composites may have trapped the paraffin molecules during melting process thus prevent it from leaking, as illustrated in Fig. 5.

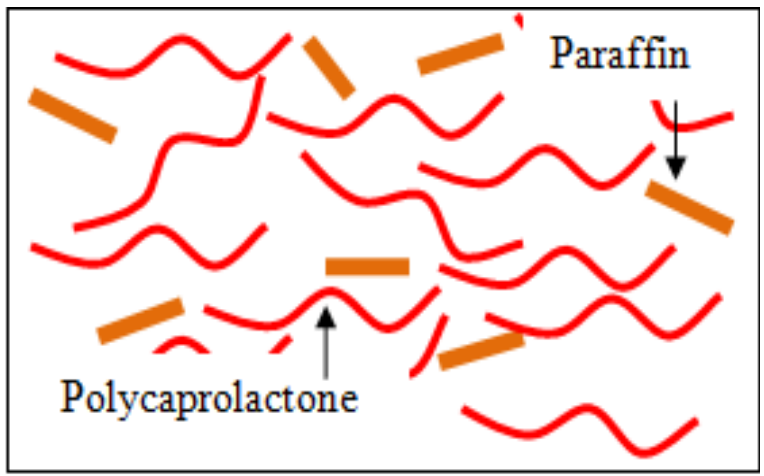

Fig. 5. Paraffin molecules in PCL matrix

\section{Conclusions}

The leakage test results indicated that by incorporating polycaprolactone (PCL) into paraffin phase, the leakage problem that associated to the pure paraffin can be prevented. The leakage characteristic of the composites was somehow related to the heat of fusion and melting temperatures of the composites. The main advantage of these composites is that its can maintain the shape and prevent paraffin leakage during phase change process thus can be used without extra encapsulation because the paraffin has been encapsulated in the polymer matrix structure. These form-stable PCMs would potentially become novel candidates of PCM for thermal energy storage applications.

The authors would like to thank Universiti Teknikal Malaysia Melaka and Ministry of Higher Education under Short Term Grant number: PJP/2014/FTK(12A)/S01322 for funding this project.

\section{References}

1. I. Dincer, M.A. Rosen, Thermal energy storage: systems and application (John Wiley \& Sons, Canada, 2012)

2. K. Pielichowska, K. Pielichowski, Prog. Mater. Sci., 65, 67 (2014)

3. A. Sharma, V.V. Tyagi, C.R. Chen, D. Budhi, Renewable and Sustainable Energy Reviews, 12, 318 (2009)

4. D. Zhou, C.Y. Zhao, Y. Tian, Appl Energy, 92, 593 (2012)

5. F. Chen, M. Wolcott, Solar Energy Materials and Solar Cells, 137, 79 (2015)

6. A. Sari, Energy Conversion and Management, 45, 2033 (2004)

7. H. Yasuda, M.S. Aludin, N. Kitamura, M. Tanabe, H. Shirahama, Macromolecules, 32, 6047 (1999) 